\title{
Depuración del lactato y mortalidad en residentes de la gran altitud con trauma grave
}

\section{Lactate clearance and mortality in high altitude residents with severe trauma}

\section{RESUMEN}

Introducción: Diversos estudios reportan que la depuración del lactato está asociado a la mortalidad en los pacientes críticos. Se describe la relación entre la depuración del lactato y la normalización del lactato con la mortalidad en los pacientes residentes de la gran altitud con trauma grave. Por lo cual es importante conocer la mortalidad de los pacientes con trauma grave en la altitud y la distribución lesional del trauma grave en la altitud. Material y Métodos: Estudio de cohorte, retrospectivo, realizado en una unidad de cuidados intensivos a 4,150 "msnm" en pacientes que ingresaron por trauma grave. Los criterios de inclusión fueron: a) Diagnóstico de trauma grave. b) Injury severity score mayor a 16. c) Presencia del resultado del análisis del lactato al ingreso y a las 6 horas en $\mathrm{UCI}$ y d) Residente de la altitud desde los 12 años. Se excluyeron los pacientes con historias clínicas incompletas y no legibles. Resultados: Se incluyeron 160 pacientes, Mortalidad del 15\%, 65\% de las lesiones fueron por trauma encefálico grave. En el grupo de supervivientes $(n=136)$, la depuración de lactato fue del $52,27 \%$ y en el grupo de fallecidos fue $21,38 \%$. En relación a la normalización (lactato< 2 $\mathrm{mmol} / \mathrm{L}$ ), el grupo de supervivientes tuvo $47 \%$ frente a $1 \%$ de los fallecidos. Conclusiones: La depuración de lactato y la normalización del lactato a las 6 seis horas son factores de protección para disminuir la mortalidad por trauma grave. La mortalidad por trauma grave es $15 \%$. El trauma encefálico grave fue la lesión más frecuente.

Palabras Clave: Altitud; Lactato; Trauma; Mortalidad(Fuente: DeCSBIREME).

\section{ABSTRACT}

Background: Several studies report that lactate clearance is associated with mortality in critically ill patients. Objectives: To describe the relationship between lactate clearance and lactate normalization with mortality in high-altitude resident patients with severe trauma. To know the mortality of patients with severe trauma at altitude. Know the injury distribution of severe trauma at altitude. Material and Methods: Epidemiological, observational, analytical, cohort, retrospective study, carried out in an intensive care unit located at 4,150 "masl" in patients admitted for severe trauma. The

\section{FILIACIÓN}

1. Hospital Corazón de Jesús - Centro de Trauma, Unidad de Medicina Critica y Terapia Intensiva, El Alto, Bolivia.

2. Seguro Social de Salud (ESSALUD), Hospital Nacional Ramiro Priale Priale, Servicio de Cuidados Intensivos e Intermedios, Huancayo, Perú.

3. Hospital Corazón de Jesús - Centro de Trauma -Servicio de Emergencia, El Alto, Bolivia.

4. Universidad de San Martín de Porres (USMP), Facultad de Medicina Humana, Centro de Investigación de Medicina en la Altura (CIMA), Huancayo, Perú.

a. Médico especialista en medicina intensiva.

b. Médico especialista en medicina de emergencia.

c. Médico especialista en cirugía general.

\section{ORCID}

1. Antonio Viruez-Soto / 0000-0002-0579-5186

2. Amilcar Tinoco-Solórzano / 0000-0002-4609-3455

3. Jorge Jiris-Quinteros / 0000-0003-4138-9641

4. Jorge Antezana-Aramayo / 0000-0003-0357-2883

5. Félix Cáceres-Flores / 0000-0003-0539-6502

\section{CORRESPONDENCIA}

Amílcar TINOCO-SOLÓRZANO

Dirección: Calle Guido 325, Huancayo, Junín-Perú Teléfono: 952991010

EMAIL

amilcartinoco@gmail.com

\section{CONFLICTOS DE INTERÉS}

Los autores niegan conflictos de interés.

\section{FINANCIAMIENTO}

Autofinanciamiento.

\section{REVISIÓN DE PARES}

Recibido: 15/07/2021

Aceptado: 15/09/2021

\section{COMO CITAR}

Viruez-Soto, A., Tinoco-Solórzano, A., Jiris-Quinteros, J., Antezana-Aramayo, J., \& Cáceres-Flores, F. Depuración del lactato y mortalidad en residentes de la gran altitud con trauma grave. y mortalidad en residentes de la gran altitud con trauma grave. A s e n jo, $2021,14(3), 291-296$. https://doi.org/10.35434/rcmhnaaa.2021.143.1247

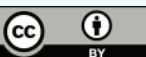

Esta obra está bajo una Licencia Creative Commons Atribución 4.0 Internacional.

Versión Impresa: ISSN: 2225-5109

Versión Electrónica: ISSN: 2227-4731

Cross Ref. DOI: $10.35434 / \mathrm{rcmhnaaa}$

OJS: https://cmhnaaa.org.pe/ojs 
inclusion criteria were: a) Diagnosis of severe trauma. b) Injury severity score greater than 16. c) Presence of the result of the lactate analysis at admission and at 6 hours in the ICU and d) Resident of the altitude since the age of 12. Patients with incomplete and unreadable medical records were excluded. Results: 160 patients were included, mortality of $15 \%, 65 \%$ of the injuries were due to severe brain trauma. In the group of survivors (136), lactate clearance was $52.27 \%$ and in the group of deceased it was $21.38 \%$. In relation to normalization (lactate $<2 \mathrm{mmol} / \mathrm{L}$ ), the group of survivors had $47 \%$ compared to $1 \%$ of the deceased. Conclusions: Lactate clearance and lactate normalization at 6 hours are protective factors to decrease mortality from severe trauma. Mortality from severe trauma is $15 \%$. Severe head trauma was the most frequent injury.

Keywords: Altitude; Lactic Acid; Severe Trauma; Mortality. (Source: DeCS-BIREME).

\section{INTRODUCCIÓN}

Definimos trauma grave a toda lesión causada por fuerza externa, que pone en peligro la vida o atenta contra alguna de las funciones vitales ${ }^{(1-3)}$. Una escala "Injury severity score" mayor a 16 también es útil para definirlo ${ }^{(4)}$. La mortalidad del trauma grave en cuidados intensivos es del $12,3 \%$ y la hospitalaria es del $16 \%{ }^{(5)}$. Aunque la distribución lesional es variable, los patrones más frecuentes son trauma craneal grave (33-47\%), trauma torácico (18-35\%), trauma de extremidades (15-26\%) y trauma abdominal $(8-17 \%)^{(1)}$.

El lactato es producido en el organismo humano a partir de la reducción de piruvato por medio de la enzima lactato deshidrogenasa, en estados fisiológicos no suele existir una acumulación de lactato ya que ésta vía metabólica es empleada únicamente para el $10 \%$ de piruvato. En un adulto sano los niveles sanguíneos normales de lactato son menores de $2 \mathrm{mmol} / \mathrm{L}$. En situaciones de hipoperfusión e hipoxia, el piruvato se acumula rápidamente y su metabolismo se desvía casi exclusivamente a la producción de lactato, por lo que el lactato intracelular se incrementa de forma exponencial para ser finalmente expulsado al medio extracelular. Por este motivo es considerado un biomarcador de hipoperfusión celular ${ }^{(6-10)}$.

Un lactato mayor de $4 \mathrm{mmol} / \mathrm{L}$ al ingreso a la unidad de cuidados intensivos se asocia a una mayor mortalidad, sin embargo, esta variable es estática por lo que actualmente se ha propuesto la depuración de lactato, como medida de tendencia durante el tratamiento de pacientes en estado crítico, habiendo sido estudiado de forma amplia en pacientes con trauma, sepsis y cirugía cardiaca ${ }^{(11-13)}$. La depuración de lactato es definida como el porcentaje de disminución de lactato y la normalización del lactato es el retorno de este a valores normales, ambos en un tiempo de seis horas ${ }^{(14,15)}$. La fórmula para calcular la depuración del lactato resulta de: [(Lactato a la admisión - Lactato a las 6h) / Lactato a la admisión] $\times 100^{(16)}$.

Toda población que se encuentra por encima de los 3,500 msnm está en la gran altitud y se considera residente si tiene una permanencia constante de un año como mínimo. Podemos tener residentes nativos o inmigrantes ${ }^{(17)}$. Trabajos previos afirman que la producción y acumulación de lactato frente al ejercicio es menor en los nativos de la altitud que en individuos nativos del nivel del mar, lo cual podría estar relacionado a una glucemia menor, una mayor concentración de glucosa intracelular, así como una utilización más eficiente de glucosa por los tejidos extrahepáticos, en individuos nativos de la altitud ${ }^{(16,18,19)}$.
Seis estudios que relacionan los valores de lactato con la mortalidad en pacientes críticos que ingresaron a cuidados intensivos por trauma a diferentes niveles de altitud encontraron que el valor de lactato mayor a $2 \mathrm{mmol} / \mathrm{l}$ estaba relacionado a mayor mortalidad y que los valores de la depuración de lactato era menor en los pacientes fallecidos. Merino et al (2010) en España a nivel del mar en 21 pacientes con trauma encefálico moderado o grave encontró que los valores elevados de lactato $(2,49 \mathrm{mmol} / \mathrm{L})$ era un hallazgo frecuente pero estos no se correlacionaron con episodios exclusivos de hipoxia tisular, ya que los niveles de oxígeno estaban dentro del rango de normalidad $(\mathrm{PtiO} 2>15 \mathrm{mmHg})$ en más del $80 \%$ de los $\operatorname{casos}^{(20)}$.

Freitas et al (2015) en Brasil a nivel del mar en 117 pacientes con politrauma encontró un lactato de $2,17 \mathrm{mmol} / \mathrm{L}$ en el grupo que fallecido y de $2,06 \mathrm{mmol} / \mathrm{L}$ en los supervivientes $(p=0,168)^{(16)}$. Marie-Alix Régnier et al (2012) en Francia a 1,313 msnm en 586 pacientes con traumatismos encontró que la depuración del lactato a las $2 \mathrm{~h}$ es una variable que debe valorarse como objetivo en la reanimación ${ }^{(19)}$. Pineda-Garcés et al (2018) en Colombia a 1,495 msnm en 130 pacientes con trauma penetrante grave encontró que la depuración de lactato a las 6 horas en los supervivientes fue de $38,3 \%$ y de $24,6 \%$ en los fallecidos ( $p=0,1585)$. El lactato a las 6 horas de los supervivientes fue de $2,33^{(21)}$. Oliveros-Rodríguez et al (2017) en Colombia a 2,654 msnm en 233 pacientes con politrauma encontró que el valor del lactato por encima de 2,35 a las 24 horas se asocia a mayor mortalidad ${ }^{(22)}$. Viruez et al (2019) en Bolivia a 4,150 msnm en 250 pacientes críticos encontró que la depuración de lactato a las 6 horas de los supervivientes disminuyo en $26 \%$ mientras que en los fallecidos se incrementó en $21 \%$. En los supervivientes el lactato a las $6 \mathrm{~h}$ fue de $2,29(\mathrm{p}<0,05)$ En el subgrupo asociado a trauma encontró que la depuración en los supervivientes disminuyo $20 \%$ mientras que en los fallecidos se incrementó en $16 \%(p<0,05)^{(23)}$.

El objetivo de la presente investigación es: Describir la relación entre la depuración del lactato y la mortalidad en los pacientes residentes de la gran altitud con trauma grave, relacionando la normalización del lactato y la mortalidad, determinando la mortalidad de los pacientes con trauma grave y la distribución lesional del trauma grave en la altitud.

\section{MATERIAL Y METODO}

Estudio de cohorte retrospectivo. Realizado en la unidad de cuidados intensivos del "Centro de Trauma - Hospital Corazón de Jesús" ubicado en la ciudad de El Alto (La Paz, Bolivia) a $4,150 \mathrm{msnm}^{(17)}$. Se analizaron la totalidad de historias clínicas 
de los pacientes que ingresaron con trauma grave del 01 de marzo del 2017 al 31 de marzo del 2019.

Los criterios de inclusión fueron a) diagnóstico de trauma grave b) Injury severity score mayor a $16 \mathrm{c}$ ) Presencia del resultado del análisis del lactato al ingreso y a las 6 horas en $\mathrm{UCl}$ y d) Residente de la altitud desde los 12 años. Se excluyeron los pacientes con historias clínicas incompletas y no legibles.

El procedimiento de recolección de la muestra se inició con la identificación y confirmación de los casos con el diagnostico de trauma grave, Se midió el valor del lactato al ingreso a la "UCI" y a las 6 horas para determinar la depuración y la normalización del lactato. Luego se hizo el seguimiento para dividir a la muestra en supervivientes y fallecidos. Para el estudio del lactato se utilizó el equipo de gasometría arterial ABL 800 FLEX de Radiometer.

El tamaño de la muestra estudiada corresponde a la totalidad de pacientes que ingresaron en el periodo de estudio con el diagnostico de trauma grave. Se realizo un muestreo no probabilístico de casos consecutivos.

En el análisis estadístico descriptivo univariado; Se verifico la normalidad de las variables cuantitativas numéricas a estudiar usando shapiro wilks. Las variables con normalidad se analizaron con media y desviación estándar, las variables sin normalidad se analizaron con mediana y rangos. Las variables cualitativas categóricas fueron analizadas utilizando frecuencias y porcentajes. En el análisis estadístico bivariado se determinará la diferencia estadísticamente significativa $(p<0,05)$, en el caso de relacionar una variable independiente cualitativa con una variable dependiente cualitativo dicotómico se usará Mc Nemor, y si se relaciona una variable independiente cuantitativo no normal con una variable dependiente cualitativo dicotómico se usará Wilcoxon. Para determinar la magnitud de la asociacion determinaremos el OR con un Intervalo de confianza 95\%. El analisis estadistico multivariado ajustando las variables confusoras para determinar que variables son protectoras o de riesgo se utilizara regresion logistica multiple. El programa estadístico utilizado fue stata 14 oficial.

Los sesgos de selección se controlaron utilizando un "método de precisión". Los sesgos de información o medición, se controlaron utilizando un "método de estandarización" La presencia de sesgos de confusión se evitó utilizando un "método de restricción".

El estudio no genero riesgos en los pacientes porque se basó en el análisis retrospectivo de historias clínicas codificadas que aseguraron la confidencialidad y anonimato de los sujetos que ingresaron al estudio. Para la ejecución de la investigación se obtuvieron los permisos institucionales. El comité de ética del Centro de Trauma - Hospital Corazón de Jesús aprobó el estudio en febrero 2020 mediante carta dirigida al investigador principal. El presente estudio fue autofinanciado por los autores.

\section{RESULTADOS}

Se estudiaron 160 pacientes con diagnóstico de trauma grave, 75,6\% $(n=121)$ eran de sexo masculino, su mediana de edad fue 40 años, $65 \%$ de la lesión fue el trauma encefálico grave. La mortalidad fue del $15 \%$. La mediana de la Depuración de lactato a las 6 horas fue de 50,5\%. 30\% de los

Tabla 1. Características y relación entre la mortalidad y la depuración del lactato en pacientes con trauma grave residentes de la gran altitud.

\begin{tabular}{|c|c|c|c|c|c|c|c|}
\hline & \multirow{2}{*}{\multicolumn{2}{|c|}{$\begin{array}{c}\text { TOTAL } \\
160\end{array}$}} & \multirow{2}{*}{\multicolumn{2}{|c|}{$\begin{array}{l}\text { SUPERVIVENTE } \\
\qquad \mathrm{n}=136\end{array}$}} & \multicolumn{2}{|c|}{ FALLECIDO } & \multirow{3}{*}{ Valor $\mathrm{p}<0.05$} \\
\hline & & & & & & 24 & \\
\hline & $(\#)$ & (\%) & $(\#)$ & (\%) & $(\#)$ & (\%) & \\
\hline Edad & $40\left(^{*}\right)$ & $13-89\left(^{* *}\right)$ & $38\left(^{*}\right)$ & $13-85\left(^{* *}\right)$ & $54,5\left(^{*}\right)$ & $17-89\left(^{* *}\right)$ & $0,010(i)$ \\
\hline \multicolumn{8}{|l|}{ Sexo } \\
\hline Masculino & 121 & 75,63 & 100 & 73,53 & 3 & 12,5 & 0,142 \\
\hline Femenino & 39 & 24,38 & 36 & 26,47 & 21 & 87,5 & \\
\hline Horas "UCl" & $120\left(^{*}\right)$ & $2-384\left(^{* *}\right)$ & $120\left(^{*}\right)$ & $24-528\left({ }^{* *}\right)$ & $47\left(^{*}\right)$ & $1-312\left({ }^{* *}\right)$ & $<0,001$ \\
\hline TEC Grave & 104 & 65 & 85 & 62,5 & 19 & 79,17 & 0,115 \\
\hline Tx Facial & 9 & 5,63 & 7 & 5,15 & 2 & 8,33 & 0,532 \\
\hline Tx Medular & 7 & 4,38 & 5 & 3,68 & 2 & 8,33 & 0,304 \\
\hline Tx Extremidades & 16 & 10 & 15 & 11,03 & 1 & 4,17 & 0,302 \\
\hline Tx Torax & 45 & 28,13 & 39 & 28,68 & 6 & 25 & 0,712 \\
\hline Ins. respiratorio agudo & 54 & 33,75 & 48 & 35,29 & 6 & 25 & 0,325 \\
\hline Tx Abdominal & 22 & 13,75 & 19 & 13,97 & 3 & 12,5 & 0,847 \\
\hline Tx Pelvico & 3 & 1,88 & 3 & 2,21 & 0 & 0 & 0,463 \\
\hline Shock hemorragico & 34 & 21,25 & 26 & 19,12 & 8 & 33,33 & 0,117 \\
\hline \multicolumn{8}{|l|}{ Lactato } \\
\hline Lactato inicial (ingreso) & $5,4\left(^{*}\right)$ & $1,8-12(* *)$ & $5,2\left({ }^{*}\right)$ & $1,8-12\left({ }^{* *}\right)$ & $6,35\left(^{*}\right)$ & $1,8-11\left({ }^{* *}\right)$ & $0,023(i)$ \\
\hline Lactato $>2 \mathrm{mmol} / \mathrm{L}$ (ingreso) & 157 & 98,13 & 134 & 98,53 & 23 & 95,83 & 0,388 \\
\hline \multicolumn{8}{|l|}{ Depuracion del lactato } \\
\hline Depuracion del lactato (\#) & $2,3\left({ }^{*}\right)$ & $-2,4-9\left(^{* *}\right)$ & $2,4\left(^{*}\right)$ & $0,3-9(* *)$ & $1,5\left(^{*}\right)$ & $0,3-4,6\left(^{* *}\right)$ & $<0,001(i)$ \\
\hline Depuracion del lactato (\%) & $50,55\left({ }^{*}\right)$ & $-99,91-81,25\left(^{* *}\right)$ & $52,27\left(^{*}\right)$ & $5,71-81,25\left({ }^{* *}\right)$ & $21,38\left({ }^{*}\right)$ & $-133,33-65,91(* *)$ & $<0,001(\mathrm{i})$ \\
\hline \multicolumn{8}{|l|}{ Normalizacion del lactato } \\
\hline Lactato Final (6 horas) & $2,2\left(^{*}\right)$ & $0,8-9,4(* *)$ & $2,2\left(^{*}\right)$ & $0,8-6,6\left({ }^{* *}\right)$ & $4,9\left(^{*}\right)$ & $1,5-11,2\left({ }^{* *}\right)$ & $<0,001(i)$ \\
\hline Lactato <2mmol/L (6 horas) & 48 & 30 & 47 & 34,56 & 1 & 4,17 & 0,003 \\
\hline
\end{tabular}

$\left(^{*}\right)$ Mediana $\left({ }^{* *}\right)$ Rango (i) Wilcoxon, Tx: Trauma, TEC: Trauma encefalico, Fx. Fractura, Ls: Lesion. 
pacientes normalizo el lactato a las 6 horas. En el Análisis bivariado, los principales hallazgos fueron que las variables de edad, horas "Uci", lactato inicial, depuración del lactato, lactato final y la normalización del lactato tuvieron una diferencia estadísticamente significativa al asociarse con la mortalidad (Tabla 1)

Tabla 2. relacion entre la mortalidad y la normalizacion del lactato en pacientes con trauma grave residentes de la gran altitud.

\begin{tabular}{|c|c|c|c|c|c|}
\hline & SUPERVIVIENTE & FALLECIDO & \multicolumn{2}{|c|}{ ASOCIACION } & \multirow[b]{2}{*}{ IC (95\%) } \\
\hline & $\#(\%)$ & $\#(\%)$ & $\mathrm{CHI}(\mathrm{P}<0.05)$ & RR & \\
\hline \multicolumn{6}{|c|}{ Lactato al ingreso } \\
\hline Menor de 2 & $2(1.47 \%)$ & $1(4.17 \%)$ & 0.39 & 0.44 & $0.08-2.28$ \\
\hline Mayor de 2 & 134 ( $98.53 \%)$ & $23(95.83 \%)$ & & & \\
\hline \multicolumn{6}{|c|}{ Lactato a las 6 horas } \\
\hline Mayor de 2 & $89(65.44 \%)$ & $23(95.83 \%)$ & 0 & 0.1 & $0.01-0.73$ \\
\hline Menor de 2 & 47 ( $34.56 \%)$ & 1 ( $4.17 \%)$ & & & \\
\hline
\end{tabular}

Encontramos asociación significativa que indica que el Lactato menor de 2 a las 6 horas es un factor de protección en relación a la mortalidad (Tabla 2 ).

El analisis multivariado utilizando regresion logistica multiple ajustado por las variables confusoras de sexo, edad, tec grave, trauma facial, trauma medular, trauma extremidades, trauma tórax y trauma abdomen muestran que la edad ( $R R=1.03, I C=1.005-1.061)$ y el lactato final $(R R=2.41, I C=1.639-3.558)$ son factores de riesgo para elevar la mortalidad. Son factores de protección para disminución de la mortalidad las Horas "UCl" (RR=0.98, IC=0.974-0.994), depuración de lactato $(\mathrm{RR}=0.46, \mathrm{IC}=0.309-0.690)$ y la normalización del Lactato ( $R R=0.06, I C=0.007-0.476)$. (Tabla $3)$.

\section{DISCUSIÓN}

Los hallazgos principales muestran que a) La mortalidad y la distribución de la lesión en el trauma grave en la altitud es similar a lo reportado a nivel mundial b) Que la depuración y la normalización del lactato a las seis horas son factores de protección para la disminución de la mortalidad en el trauma grave.

En la altitud se ha reportado la "Paradoja del lactato" que indica que durante la exposición aguda a la hipoxia hipobarica hay una mayor acumulación de lactato en sangre en comparación con el mar nivel, pero después de una exposición continua de más de 6 semanas este fenómeno metabólico transitorio se revierte ${ }^{(24-26)}$, por lo tanto los residentes de la altitud deben tener niveles de lactato normal menor a $2 \mathrm{mmol} / \mathrm{L}$, por lo cual este valor también debe ser el objetivo en la normalización.

La mortalidad encontrada en la altitud por trauma grave fue del $15 \%$ similar a cualquier otra UCI polivalente del mundo ${ }^{(5)}$. La distribución lesional del trauma grave en la altitud también es similar a lo reportado a nivel mundial. Las lesiones más frecuentes fueron 1) trauma encefálico grave y estuvo relacionado a hematoma subdural y epidural. 2) trauma tórax, $54 \%$ de estos presentaron insuficiencia respiratoria aguda. c) trauma abdominal asociado a lesión hepática y de intestinos y d) Trauma de extremidades con fractura de tibia y fémur ${ }^{(1,27,28)}$. Esto indica que la altitud no es un factor que cambie la epidemiologia del trauma grave.

Nuestros resultados indican que el valor del lactato inicial así este sea mayor de $2 \mathrm{mmol} / \mathrm{L}$ al ingreso a UCI no influyen en la mortalidad. También hemos encontrado que la edad y el valor del lactato a las seis horas por encima de $2 \mathrm{mmol} / \mathrm{L}$ son factores de riesgo para elevar la mortalidad y que una menor cantidad de horas $\mathrm{UCl}$, la depuración del lactato y la normalización del lactato $<2 \mathrm{mmol} / \mathrm{L}$ a las 6 horas son

Tabla 3. Regresion logística múltiple de los factores de riesgo y protección relacionados con la mortalidad del trauma grave.

\begin{tabular}{|c|c|c|c|c|c|c|}
\hline MORTALIDAD & $\mathrm{RR}\left({ }^{*}\right)$ & IC(95\%)Li-LS & $p<0.05$ & $\operatorname{RR}\left({ }^{* *}\right)$ & IC(95\%) Li-Ls & $p<0.05$ \\
\hline Sexo Masculino & 2,52 & $0,71-8,96$ & 0,153 & 2,86 & $0,69-11,76$ & 0,146 \\
\hline Edad & 1,03 & $1,01-1,06$ & 0,008 & 1,03 & $1,01-1,06$ & 0,019 \\
\hline Horas "UCl" & 0,98 & $0,97-0,99$ & $<0,001$ & 0,98 & $0,97-0,99$ & 0,002 \\
\hline TEC Grave & 2,28 & $0,80-6,48$ & 0,122 & 4,43 & $0,80-24,59$ & 0,089 \\
\hline TX Facial & 1,68 & $0,33-8,60$ & 0,536 & 2,41 & $0,25-23,43$ & 0,449 \\
\hline TX Medular & 2,38 & $0,43-13,05$ & 0,317 & 7,05 & $0,83-60,02$ & 0,074 \\
\hline TX Torax & 0,83 & $0,31-2,24$ & 0,712 & 1,92 & $0,40-9,19$ & 0,416 \\
\hline TX Abdominal & 0,88 & $0,24-3,24$ & 0,847 & 1,31 & $0,22-7,69$ & 0,765 \\
\hline \multicolumn{7}{|l|}{ Lactato } \\
\hline Lactato inicial (ingreso) & 1,18 & $0,97-1,43$ & 0,089 & 1,04 & $0,81-1,32$ & 0,774 \\
\hline Lactato $>2 \mathrm{mmol} / \mathrm{L}$ (ingreso) & 0,34 & $0,03-3,94$ & 0,391 & 0,38 & $0,03-5,02$ & 0,464 \\
\hline \multicolumn{7}{|l|}{ Depuracion del lactato } \\
\hline Depuracion del lactato (\#) & 0,5 & $0,35-0,70$ & $<0,001$ & 0,46 & $0,31-0,69$ & $<0,001$ \\
\hline Lactato final (6 horas) & 2,04 & $1,53-2,71$ & $<0,001$ & 2,42 & $1,64-3,56$ & $<0,001$ \\
\hline Lactato <2mmol/L (6 horas) & 0,08 & $0,01-0,63$ & 0,016 & 0,06 & $0,01-0,48$ & 0,008 \\
\hline
\end{tabular}

Tx: Trauma, TEC: Trauma encefalico, Fx. Fractura, Ls: Lesion. $\left(^{*}\right)$ RR Crudo "REGRESION LOGISTICA SIMPLE", (**) RR Ajustado "REGRESION LOGISTICA MULTIPLE" 
factores de protección para disminuir la mortalidad.

Encontramos que el grupo se supervivientes tuvo una depuración a las seis horas del $52,27 \%$ y del $21,38 \%$ en los fallecidos $(p<0,05)$ y que el $47 \%$ de los pacientes del grupo de supervivientes normalizo su lactato $(<2 \mathrm{mmol} / \mathrm{L})$ y de $1 \%$ en los fallecidos $(p<0,05)$. Resultados anteriores en pacientes críticos asociados a trauma también han encontrado que la depuración del lactato de los supervivientes (20 a 38,6\%) es mayor al de los fallecidos ${ }^{(21,23)}$. En relación a la normalización los estudios de críticos con trauma también se encontró menores valores de lactato a las 6 horas en el grupo supervivientes $(2,06$ a $2,33 \mathrm{mmol} / \mathrm{L})$ que, en los fallecidos, pero sin llegar a valores de normalización. También existen estudios que relacionan la depuración de lactato a las 2, 6, 24 horas con la mortalidad en pacientes críticos por sepsis y críticos quirúrgicos que demostró que los supervivientes tenían una mayor depuración que en los fallecidos ${ }^{(11,12,29-32)}$ En relación a la normalización del lactato en un nivel de 1,76 $\mathrm{mmol} / \mathrm{L}$ es un poderoso indicador durante la reanimación ${ }^{(29,32)}$. por esto, si el tratamiento hemodinámico intensivo logra normalizar los valores de lactato en el menor tiempo posible, el riesgo de mortalidad puede verse disminuido ${ }^{(33,34)}$. La resucitación cuantitativa en pacientes críticamente enfermos comprende intervenciones hemodinámicas tales como la expansión del volumen intravascular y soporte vasoactivo buscando alcanzar parámetros fisiológicos en el menor tiempo posible ${ }^{(35-39)}$.

El tamaño de la muestra estudiada a pesar de utilizar un muestreo no probabilístico de casos consecutivos y representar a la totalidad de pacientes que ingresaron en el periodo de estudio con el diagnostico de trauma grave no fue la ideal y esto representaría la principal limitación del estudio.

En conclusión: a) La depuración de lactato a las 6 seis horas es un factor de protección para disminuir la mortalidad por trauma grave. b)) La normalización del lactato a las 6 seis horas es un factor de protección para disminuir la mortalidad por trauma grave c) La mortalidad por trauma grave es del $15 \%$. d) El trauma encefálico grave fue la lesión más frecuente. Recomendamos plantear como objetivo terapéutico durante la reanimación en pacientes críticos, la depuración de lactato poniendo énfasis en lograr su normalización a las seis horas en individuos nativos y residentes de la altitud.

\section{REFERENCIAS BIBLIOGRÁFICAS}

1. Alberdi F, García I, Atutxa L, Zabarte M, Trauma and Neurointensive Care Work Group of the SEMICYUC. Epidemiology of severe trauma. Med Intensiva. 2014;38(9):580-8

2. Infante Carbonell M, Cabrera Salazar J, Puertas Álvarez J. Morbilidad y mortalidad por traumatismo con lesión visceral. MEDISAN. 2010;14(7):968-75

3. Domínguez González E, Cisneros Domínguez C, Piña Prieto L. Mortalidad por lesiones traumáticas en pacientes hospitalizados. Rev Cuba Cir. 2014;53(4):324-35.

4. Rapsang A, Shyam D. Scoring Systems of Severity in Patients with Multiple Trauma. Cir Esp Engl Ed. 2015;93(4):213-21.

5. Chico-Fernández M, Llompart-Pou J, Guerrero-López F, SánchezCasado M, García-Sáez I, Mayor-García M, et al. Epidemiology of severe trauma in Spain. Registry of trauma in the ICU (RETRAUCI). Pilot phase. Med Intensiva. 2016;40(6):327-47.
6. Kraut J, Madias N. Lactic acidosis. N Engl J Med. 2014;371(24):230919.

7. Marik P, Bellomo R. Lactate clearance as a target of therapy in sepsis: A flawed paradigm. OACrit Care. 2013;1(1):1-6.

8. Gibot $\mathrm{S}$. On the origins of lactate during sepsis. Crit Care. 2012;16(5):151.

9. Bermúdez-Rengifo WA, Fonseca-Ruiz NJ. Utilidad del lactato en el paciente críticamente enfermo. Acta Colomb Cuid Intensivo. 2016;16(2):80-9.

10. Bakker J, Postelnicu R, Mukherjee V. Lactate: Where Are We Now? Crit Care Clin. 2020;36(1):115-24.

11. Zhang $Z, X u X$. Lactate clearance is a useful biomarker for the prediction of all-cause mortality in critically ill patients: a systematic review and meta-analysis*. Crit Care Med. 2014;42(9):2118-25.

12. Lindsay A, Xu M, Sessler D, Blackstone E, Bashour C. Lactate clearance time and concentration linked to morbidity and death in cardiac surgical patients. Ann Thorac Surg. 2013;95(2):486-92.

13. Vásquez-Tirado GA, García-Tello AV, Evangelista Montoya FE. Utilidad del lactato sérico elevado como factor pronóstico de muerte en sepsis severa | Horizonte Médico (Lima). Horiz Méd Lima. 2015;15(2):35-40.

14. Porras-García W, Ige-Afuso M, Ormea-Villavicencio A. Depuración de lactato como indicador pronóstico de mortalidad en pacientes con sepsis severa y choque séptico. Rev Soc Peru Med Interna. 2007;20(4):132-8

15. Gonzáles M, Hernando Morales C, Sanabria A. Aclaramiento de lactato como factor pronóstico en pacientes con traumatismo penetrante Revisión sistemática de la literatura. Rev Colomb Cir. 2016;31(1):3443.

16. Freitas A, Franzon O. Lactate as predictor of mortality in polytrauma. Arq Bras Cir Dig. 2015;28(3):163-6.

17. Tinoco-Solórzano A, Estrada Nieto V, Vélez-Páez J, Franco Molano D, Viruez Soto AV, Villacorta-Córdova F, et al. Medicina intensiva en la Altitud. Revision de Alcance. Intensivos. 2020;13(4):218-25.

18. Gonzales G. Metabolismo en las grandes alturas. Acta Medica. 2001;9(1):31-45.

19. Régnier M, Raux M, Le Manach Y, Asencio Y, Gaillard J, Devilliers C, et al. Prognostic significance of blood lactate and lactate clearance in trauma patients. Anesthesiology. 2012;117(6):1276-88.

20. Merino M, Sahuquillo J, Borrull A, Poca M, Riveiro M, Expósito L. ¿Es el lactato un buen indicador de hipoxia tisular?: Resultados de un estudio piloto en 21 pacientes con un traumatismo craneoencefálico. Neurocirugía. 2010;21(4):289-301.

21. Pineda-Garcés D, González-Urhan M, Morales-Uribe C. Aclaramiento de lactato en trauma penetrante grave. Rev Colomb Cir. 2018;33(4):380-9.

22. Oliveros-Rodríguez $H$, Estupiñán-López R, Rodríguez-Gómez J. Mediciones seriadas del lactato y su validez predictiva de la mortalidad temprana en los pacientes con politrauma que ingresan a la unidad de cuidado intensivo. Rev Colomb Anestesiol. 2017;45(3):17.

23. Viruez-Soto J, Ibáñez-Velasco B, Jiménez-Torres F, Briones-Garduño $J$, Díaz de León-Ponce M, Vera-Carrasco O. Depuración de lactato a gran altitud. Cuad Hosp Clínicas. 2019;60(1):32-6.

24. Lundby C, Saltin B, Hall G. The «lactate paradox», evidence for a transient change in the course of acclimatization to severe hypoxia in lowlanders. Acta Physiol Scand. 2000;170(4):265-9.

25. Hochachka PW, Beatty CL, Trump ME, McKenzie DC, Matheson GO. The Lactate Paradox in Human High-Altitude Physiological Performance. News Physiol Sci. 2002;17:122-6.

26. Van Hall G, Lundby C, Araoz M, Calbet J a. L, Sander M, Saltin B. The lactate paradox revisited in lowlanders during acclimatization to $4100 \mathrm{~m}$ and in high-altitude natives. J Physiol. 2009;587(Pt 5):111729.

27. Montalvo M, Vélez J, Jara F, Velarde G, Vélez P, Juan P. Aclaramiento del lactato: revisión de la literatura. Metro Cienc. 2018;26(1):39-42.

28. Park YH, Ryu DH, Lee BK, Lee DH. The association between the initial lactate level and need for massive transfusion in severe trauma patients with and without traumatic brain injury. Acute Crit Care. 2019;34(4):255-62

29. Bhat S, Swenson K, Francis M, Wira C. Lactate Clearance Predicts Survival Among Patients in the Emergency Department with Severe Sepsis. West J Emerg Med. 2015;16(7):1118-26.

30. Cardinal Fernández $\mathrm{P}$, Olano $\mathrm{E}$, Acosta $\mathrm{C}$, Bertullo $\mathrm{H}$, Albornoz $\mathrm{H}$, Bagnulo $\mathrm{H}$. Valor pronóstico del aclaramiento de lactato en las 
primeras $6 \mathrm{~h}$ de evolución en medicina intensiva. Med Intensiva. 2009;33(4):166-70.

31. Walker C, Griffith D, Gray A, Datta D, Hay A. Early lactate clearance in septic patients with elevated lactate levels admitted from the emergency department to intensive care: time to aim higher? J Crit Care. 2013;28(5):832-7.

32. Jones A. Lactate clearance for assessing response to resuscitation in severe sepsis. Acad Emerg Med Off J Soc Acad Emerg Med. 2013;20(8):844-7.

33. Andersen L, Mackenhauer J, Roberts J, Berg K, Cocchi M, Donnino M Etiology and therapeutic approach to elevated lactate levels. Mayo Clin Proc. 2013;88(10):1127-40.

34. Glenn H, Regueira T, Bruhn A, Castro R, Rovegno M, Fuentealba A, et al. Relationship of systemic, hepatosplanchnic, and microcirculatory perfusion parameters with 6-hour lactate clearance in hyperdynamic septic shock patients: an acute, clinical-physiological, pilot study. Ann Intensive Care. 2012;2(1):1-9.
35. Puskarich M, Trzeciak S, Shapiro N, Arnold R, Heffner A, Kline J, et al. Prognostic value and agreement of achieving lactate clearance or central venous oxygen saturation goals during early sepsis resuscitation. Acad Emerg Med Off J Soc Acad Emerg Med. 2012;19(3):252-8.

36. He $\mathrm{H}$, Liu $\mathrm{D}$, Long $\mathrm{Y}$, Wang $\mathrm{X}$. High central venous-to-arterial $\mathrm{CO} 2$ difference/arterial-central venous $\mathrm{O2}$ difference ratio is associated with poor lactate clearance in septic patients after resuscitation. $J$ Crit Care. 2016;31(1):76-81.

37. Jones A. Point: Should Lactate Clearance Be Substituted for Centra Venous Oxygen Saturation as Goals of Early Severe Sepsis and Septic Shock Therapy? Yes. Chest. 2011;140(6):1406-8.

38. Fuller $B$, Dellinger R. Lactate as a hemodynamic marker in the critically ill. Curr Opin Crit Care. 2012;18(3):267-72

39. Pino RM, Singh J. Appropriate Clinical Use of Lactate Measurements. Anesthesiology. 2021;134(4):637-44. 